\title{
Las uniones homosexuales en España. Una caracterización sociodemográfica a partir del censo de $2001^{1}$
}

\author{
Clara Cortina* \\ Anna Cabré**
}

${ }^{*}$ Consejo Superior de Investigaciones Científicas

clara.cortina@cchs.csic.es

${ }^{* *}$ Centre d'Estudis Demogràfics

anna.cabre@uab.cat

Recibido: 10-10-2007

Aceptado: 11-06-2008

\begin{abstract}
En el contexto de la reciente legalización del matrimonio homosexual en España, sorprende la ausencia de análisis empíricos sobre dicho tipo de uniones en este país. Con el fin de contribuir a salvar este vacío, el presente artículo estudia a los cónyuges y a las uniones homosexuales en España a partir del censo de 2001. Se analizan la edad, el sexo, el nivel de instrucción y la nacionalidad de los miembros de la pareja, así como la composición de las uniones respecto a estas mismas variables. Los resultados descubren diferencias significativas según el tipo de unión y permiten destacar el mayor grado de heterogamia de las uniones homosexuales.
\end{abstract}

Palabras clave: uniones homosexuales, censo de 2001, homogamia, matrimonio.

Abstract Same-sex unions in Spain. The Sociodemographic Profile from the 2001 census data

Same-sex marriage has recently become legalized in Spain in a context of scarce empirical research on same-sex partnerships. This article aims to contribute to this field by using the 2001 census data to describe the demographics of same-sex partners and partnerships. We include an overview of the demographic characteristics of the spouses and analyze the composition of same-sex partnerships in terms of age, sex, educational attainment and citizenship. The results point out that individual and union characteristics differ according to sex composition of the couple. Same-sex partnerships appear to be significantly more heterogamous than the heterosexuals ones.

Key words: same-sex partnerships, 2001 census, assortative mating, marriage.

1. Este trabajo debe considerarse producto del proyecto El impacto de la población extranjera en la formación de la pareja en España (SEJ2007-60014), subvencionado por el plan nacional I+D+I del Ministerio de Educación y Ciencia. Las autoras quieren agradecer a los doctores Pau Miret y Albert Esteve sus atentas lecturas de este artículo. Clara Cortina es investigadora del Grupo de Investigación de Dinámicas Demográficas (GIDD) y del Grupo de Estudios de Población y Sociedad (GEPS). 


\begin{tabular}{|c|c|}
\hline \multicolumn{2}{|c|}{ Sumario } \\
\hline 1. Introducción & Fuentes de datos para el estudio \\
\hline La legalización de las uniones & de las uniones disponibles en España \\
\hline homosexuales: contexto jurídico & Las uniones homosexuales en España \\
\hline $\begin{array}{r}\text { El estudio de las uniones homosexuales: } \\
\text { evidencia empírica }\end{array}$ & Referencias bibliográficas \\
\hline
\end{tabular}

\section{Introducción}

Desde el 3 de julio de 2005, día de entrada en vigor de la Ley 13/2005, por la que se modifica el código civil en materia de derecho a contraer matrimonio, se pueden celebrar en España bodas entre dos cónyuges del mismo sexo. La universalización del matrimonio en España se produce en un contexto europeo de progresiva legalización de las uniones homosexuales y pone de manifiesto el interés por conocer estas uniones desde un punto de vista sociológico y demográfico (Festy, 2006). Sin embargo, los estudios que se han llevado a cabo han topado con las dificultades de captación estadística de las uniones homosexuales. En España, hasta el momento, para abordar este análisis, sólo disponíamos del censo de población de 2001 y de encuestas específicas. El hecho de que el último censo registrara todas las uniones con independencia del sexo de sus miembros constituye un privilegio estadístico notable en el contexto europeo, al que hay que añadir la perspectiva de poder trabajar en el futuro con el registro de matrimonios, que, de acuerdo con el cambio jurídico, registra ahora bodas entre hombre y mujer, entre hombre y hombre y entre mujer y mujer.

En este contexto, en el presente trabajo, constatamos la ausencia de investigación empírica sobre las parejas homosexuales en España y nos proponemos explorar las fuentes estadísticas disponibles para su estudio. Nuestro objetivo principal es analizar dichas parejas a partir de la información proporcionada por el censo de población de 2001, centrando nuestro análisis en la caracterización sociodemográfica de los cónyuges y en la composición de las uniones, buscando resaltar las semejanzas y las diferencias existentes con las parejas heterosexuales. Nos preguntamos si los individuos que viven en pareja con una persona de su mismo sexo presentan rasgos propios y, sobre todo, si la composición de dichas uniones es más dispar que la de las heterosexuales en características como, por ejemplo, la edad, el nivel de instrucción o la nacionalidad, teniendo en cuenta que no lo son en el sexo. Es decir, el grado de heterogamia de las uniones (la disparidad entre las características de los miembros) aumenta en las parejas donde ambos cónyuges son del mismo sexo.

\section{La legalización de las uniones homosexuales: contexto jurídico}

El proceso de legalización de las uniones homosexuales en Europa se inicia durante la década de 1990 y se concreta en fórmulas distintas en cada país. 
Algunos países abren la vía al registro de las bodas homosexuales en el marco de las uniones de hecho (Noruega, Francia, Alemania), otros igualan dichas uniones a las heterosexuales, hasta el punto de considerarlas igualmente matrimonios (Suecia, Holanda, Bélgica y, recientemente, también España). Tanto en un caso como en otro, la legalización de la unión supone una ampliación de los derechos de los consortes, si bien no suelen igualarse dichos derechos a los de las parejas heterosexuales (tampoco en aquellos casos en los que se legaliza el matrimonio homosexual), según concluye Waaldijk (2005) en un amplio y riguroso estudio donde realiza una comparación de las consecuencias legales de la legalización de las uniones homosexuales en nueve países europeos.

Evidentemente, este proceso de legalización entraña importantes implicaciones en la concepción del matrimonio y la pareja, de la misma manera que la expansión del fenómeno de la cohabitación en Europa lo había hecho durante la década precedente. Resulta interesante ver como, tanto en un caso como en el otro, los científicos coinciden en señalar que el estancamiento del fenómeno de la cohabitación, por un lado, y la apertura del matrimonio a las parejas del mismo sexo, por otro, se explican precisamente por la transformación de la institución matrimonial. En el primer caso, Domingo (1997) sostiene que la capacidad innovadora de las parejas de cohabitantes en España durante los últimos años de la década de 1980 y primeros de la de 1990, no se limita a sí mismas, sino que transforma incluso la forma legal de pareja, de tal modo que las diferencias entre ambas concepciones se diluyen y el recurso a la pareja de hecho pierde sentido. Es lo que podríamos llamar morir de éxito. En el segundo caso, el sociólogo noruego Moxnes (citado por Andersson et al., 2006) considera que la legalización del matrimonio homosexual no habría resultado aceptable si, previamente, el matrimonio no se hubiera vaciado de contenido como institución y hubiera dejado de ser la vía exclusiva de llegada a la vida adulta, a la vida sexual y a la paternidad.

Como en Suiza, otro país con descentralización legislativa, antes de la reforma del Código Civil de 2005, en España se habían empezado a legalizar las uniones homosexuales de manera descentralizada, es decir, mediante legislación autonómica. Esta legislación tenía un objetivo común: establecer la posibilidad de registrar a las parejas de hecho con independencia del sexo de los cónyuges. En concreto, entre 1998 y 2005, doce de las diecisiete comunidades autónomas españolas ${ }^{2}$ habían aprobado leyes de parejas de hecho, según las

2. Cataluña: Ley 10/1998, de 15 de julio, de uniones estables de pareja; Aragón: Ley 6/1999, de 26 de marzo, relativa a parejas estables no casadas; Navarra: Ley Foral 6/2000, de 3 de julio, para la igualdad jurídica de las parejas estables; Valencia: Ley 1/2001, de 6 de abril, por la que se regulan las uniones de hecho; Baleares: Ley 18/2001, de 19 de diciembre, de parejas estables; Madrid: Ley 11/2001, de 19 de diciembre, de uniones de hecho de la Comunidad de Madrid; Asturias: Ley 4/2002, de 23 de mayo, de parejas estables del Principado de Asturias; Andalucía: Ley 5/2002, de 28 de diciembre, de parejas de hecho de Andalucía; Extremadura: Ley 5/2003, de 20 de marzo, de parejas de hecho de la comunidad autónoma de Extremadura; Canarias: Ley 5/2003, de 6 de marzo, de las parejas de hecho de la comunidad autónoma de Canarias; País Vasco: Ley 2/2003, de 7 de mayo, reguladora de las parejas de hecho; Cantabria: Ley 1/2005, de 16 de mayo, de parejas de hecho de la comunidad autónoma de Cantabria. 
cuales éstas podían registrarse sin contraer matrimonio. Dichas leyes, que difieren entre sí en los derechos que garantizan a las parejas, daban respuesta a una doble demanda social: las exigencias de cobertura de las parejas cohabitantes, por una parte, y las de las parejas homosexuales (cohabitantes también), por otra. Supusieron además, juntamente con la mayor aceptación social y las exigencias de los colectivos de gays y lesbianas, un claro elemento de presión para que el Gobierno central se decidiera a legislar sobre la materia en el año 2005 (Pichardo Galán, 2004). Sin embargo, el paso de las leyes autonómicas de parejas de hecho a la reforma del código civil por parte del Gobierno socialista supone no tan sólo un cambio de escala, sino, fundamentalmente, un cambio de lógica: en lugar de crear una fórmula ad hoc para las parejas cohabitantes de todo sexo al margen del matrimonio, se opta por universalizar el contrato matrimonial.

\section{El estudio de las uniones homosexuales: evidencia empírica}

Dadas las dificultades de captación estadística que se derivan de la invisibilidad social y administrativa de las uniones homosexuales, el interés fundamental de su estudio ha residido en conocer su incidencia, es decir, su número. Andersson et al. (2006) cifran entorno al 7\%o en Noruega y al 5\%o en Suecia el peso de las uniones homosexuales sobre el total de las que se habían realizado (a partir de los registros de uniones y matrimonios); Festy y Digoix (2004), en el 6\%o para Francia (a partir de una estimación) y en el 12\%o para Holanda (según una encuesta retrospectiva), y, finalmente, Black et al. (2000), en un $10 \%$ según los censos de los Estados Unidos. La variación entre las distintas cifras se explica, entre otras razones, por la naturaleza de las fuentes utilizadas, pero da cuenta en buena medida de la minoritaria presencia de dichas uniones. La voluntad de conocer el número de parejas homosexuales deriva del interés en cifrar la incidencia de la homosexualidad, es decir, el número de homosexuales. Sin embargo, este objetivo se enfrenta a dificultades metodológicas de mayor envergadura, empezando por la propia definición de la condición de homosexual. En este sentido, el estudio de las uniones homosexuales es más realista y parte de una definición, la de pareja estable corresidente (de hecho o de derecho), que presenta menos ambigüedades. Los investigadores también se han planteado la necesidad de ampliar el ámbito de estudio a parejas estables no residentes, una forma muy extendida entre los colectivos homosexuales (Festy y Digoix, 2004).

En lo que al estudio de las uniones homosexuales estables se refiere, se han desarrollado análisis equivalentes a los llevados a cabo para las parejas heterosexuales (si bien afrontando mayores dificultades en la obtención de información), es decir, análisis que se han interesado por la intensidad y el calendario de formación de las uniones, así como por los procesos de ruptura y por la composición de las uniones. En cada uno de estos aspectos, los análisis pioneros han tomado como punto de referencia la evidencia empírica proporcionada por las parejas heterosexuales. A medida que los estudios se han ido desa- 
rrollando, en distintos países se ha abierto también la posibilidad de realizar análisis comparativos. Nuestro estudio de las uniones homosexuales en España se centra de manera específica en una de las dimensiones señaladas: la composición.

El análisis de la composición de las parejas se ha planteado tradicionalmente en términos de homogamia y heterogamia, es decir, mediante el análisis del grado de semejanza entre los cónyuges en relación con una serie de variables adscritas o adquiridas (edad, origen, nivel de instrucción, religión, raza, profesión, nivel socioeconómico, etc.), con lo cual se cualifican las uniones entre cónyuges que comparten determinada característica de homógamas y las uniones entre cónyuges distintos de heterógamas. La distribución de las parejas según el grado de homogamia es un indicador de uso frecuente en el estudio de la composición de aquéllas, y aporta, por extensión, elementos para conocer las estrategias matrimoniales y la interacción entre grupos y categorías sociales. Los estudios llevados a cabo en distintos países y a lo largo del tiempo han puesto de manifiesto el predominio de la homogamia, pero también su variabilidad y su dependencia, tanto de factores preferenciales, es decir, del criterio individual en la selección del cónyuge, como de factores estructurales, es decir, de las condiciones de oferta de cónyuges posibles en el llamado mercado matrimonial. La homogamia aumenta en aquellos mercados en los que los efectivos se caracterizan por su homogeneidad, pero también se impone en contextos de heterogeneidad precisamente por la fuerza de atracción entre iguales («The like likes the like»).

En España, la evidencia empírica demuestra que los cónyuges se unen de forma homógama de acuerdo con la edad que tienen, sus niveles de instrucción y sus orígenes geográficos (Esteve, Cortina, Cabré, 2009; Esteve y Cortina, 2006, 2007). Concretamente, la diferencia media de edad entre los cónyuges ha decrecido en los últimos veinticinco años. Ello ha sido debido, fundamentalmente, a dos motivos: las mujeres son mayores que antes cunado se casan y, además, hoy, los hombres, a todas la edades, se casan en primeras nupcias con mujeres de una edad más cercana a la suya de lo que lo hacían hace un cuarto de siglo. Por otra parte, la pareja formada por un hombre y una mujer de nivel académico equivalente es el modelo estándar de pareja en España, si bien ha perdido fuerza durante el siglo XX, en un proceso de cambio generacional remarcable. Esta tendencia general a la reducción de los niveles de homogamia referente a la instrucción esconde diferencias significativas entre grupos educativos: los niveles de homogamia entre los universitarios aumentan significativamente de generación en generación, mientras se reducen en los grupos de educación inferior. Además, el predominio de la hipergamia femenina (la pauta según la cual las mujeres se unen mayoritariamente con hombres más instruidos que ellas) ha perdido definitivamente vigencia. Y, finalmente, en relación con el origen, los españoles y las españolas se emparejan preferentemente con otros españoles y españolas de su mismo municipio, provincia, comunidad autónoma y país. A excepción del municipio, para el resto de las escalas, la variación de los niveles de homogamia entre las cohortes es parale- 
la a la variación de la proporción de migrantes de cada cohorte. Es decir, los niveles de homogamia son más bajos en aquellas cohortes que más han migrado.

Desde la perspectiva de la composición de las parejas, las uniones homosexuales son homógamas en relación con el sexo. La evidencia de que las parejas heterosexuales tienden a la homogamia en otras características sociodemográficas nos lleva a preguntarnos si es también el caso para las parejas homosexuales o bien si, precisamente por serlo absolutamente en relación con la variable fundamental del sexo, pueden serlo menos en relación con las demás. En este sentido, Andersson et al. (2006) observan, para las parejas homosexuales suecas y noruegas, una mayor disparidad de edades entre los cónyuges, así como entre sus niveles educativos y su nacionalidad, especialmente en las parejas de hombres. También en Francia, Festy, Digoix y Garnier (2004) detectan mayores diferencias de edad entre los cónyuges en unión homosexual. En contraposición a estos estudios, no existe apenas literatura para España sobre la materia. Se trata de un tema muy poco explorado en este país, fundamentalmente debido a las deficiencias de la información estadística. A continuación, veremos con qué fuentes estadísticas contamos efectivamente.

\section{Fuentes de datos para el estudio de las uniones disponibles en España}

Para estudiar a las parejas españolas en los últimos años, existen dos fuentes estadísticas fundamentales: el censo de población de 2001 y el registro de matrimonios del movimiento natural de la población (MNP). Se trata de dos fuentes de naturaleza distinta que proporcionan datos hasta cierto punto complementarios. En el caso del censo, conocemos a todas las parejas corresidentes en España y que se declaran como tales (o a una muestra representativa de ellas en el caso de trabajar con microdatos), con independencia de que se trate de uniones de hecho o de matrimonios. En el caso del MNP, conocemos solamente aquellos matrimonios que se constituyen en España año a año. Si bien las uniones de hecho quedan, así, fuera de observación en el MNP, con él ganamos en vigencia, puesto que actualmente ya están disponibles los datos hasta 2008, mientras que el censo se queda en 2001. Otro elemento que distingue al censo en relación con el MNP es la cantidad de variables de las que dispone, que permiten una caracterización muy completa, tanto de los cónyuges como de las uniones.

Todo lo dicho vale tanto para las uniones heterosexuales como para las homosexuales. Sin embargo, hay una diferencia determinante: los matrimonios homosexuales sólo se celebran a partir de julio de 2005. Las perspectivas que abre esta fuente son esperanzadoras. Como ocurre con el registro de uniones estables de Noruega y de matrimonios de Suecia (Andersson et al., 2006), con el MNP se pueden las tasas de nupcialidad homosexual y las edades medias al matrimonio. Sin embargo, algunas posibilidades de análisis privilegiadas derivadas de la integración de distintas fuentes estadísticas que existe en estos países nórdicos, siguen siendo inimaginables en España. No es posible calcular probabilidades de ruptura ni, por consiguiente, aplicar análisis biográficos. 
Tabla 1. Parejas según tipo de unión y fuente

\begin{tabular}{lcccc}
\hline & $\begin{array}{c}\text { Parejas de } \\
\text { distinto sexo }\end{array}$ & $\begin{array}{c}\text { Parejas del } \\
\text { mismo sexo, } \\
\text { hombres }\end{array}$ & $\begin{array}{c}\text { Parejas del } \\
\text { mismo sexo, } \\
\text { mujeres }\end{array}$ & $\begin{array}{c}\text { Parejas del } \\
\text { mismo sexo } \\
\text { por mil parejas }\end{array}$ \\
\hline Censo 2001 & 9.500 .343 & 6.996 & 3.478 & 1,1 \\
Muestra 5\% hogares censo 2001 & 475.019 & 336 & 168 & 1,1 \\
Matrimonios 2005-2008 & 722.504 & 8.296 & 3.934 & 16,6 \\
\hline
\end{tabular}

Fuente: censo de población 2001 y MNP 2005.

Aquí planteamos una exploración de las parejas según el tipo de unión ${ }^{3}$ registradas en el censo población de 2001. En concreto, se contabilizan 6.996 parejas homosexuales de hombres y 3.478 de mujeres, lo que representa un 1,1\%o del total de parejas (ver tabla 1).

Ante todo, es preciso destacar que el censo de población de 2001 es el primero en España que permite la declaración de una relación conyugal de parentesco entre dos individuos del mismo sexo, característica que lo hace también pionero en Europa. Sin embargo, esta posibilidad debe ser tomada con cautela, puesto que es necesario cuestionar el ajuste de estas cifras al número real de parejas homosexuales corresidentes en España. El 1\%o de las parejas supone uno de los niveles más bajos en el contexto europeo y occidental, que, como hemos visto, se mueve entre el $5 \%$ y el $12 \%$, pero además contrasta, a nivel español, con el 16,6\% que representan los matrimonios homosexuales entre 2005 y 2008 sobre el total de matrimonios del mismo período. Aunque esta intensidad puede explicarse en parte por la respuesta coyuntural al cambio legislativo (como ocurrió con el divorcio tras su legalización en España en 1981), es razonable pensar que el censo haya subregistrado el número de parejas homosexuales. Si bien la autodeclaración ${ }^{4}$ en el censo gozaba de todas las garantías de confidencialidad estadística, es razonable pensar que no todas las parejas homosexuales se declararan como tales por prudencia, en un contexto en el que algunas de estas relaciones pueden permanecer escondidas socialmente. El censo de 2001 ofrece posibilidades adicionales para tratar de salvar los límites de la autodeclaración: a partir de las relaciones de parentesco, es posible reconstruir con precisión los hogares e identificar aquellas personas del mismo sexo que conviven sin tener relación de parentesco alguna. Una vez identificadas estas parejas de individuos, sería posible aplicar técnicas como las desarrolladas por Festy, Digoix y Garnier (2004) para el censo francés de 1999 (que no ofrecía la posibilidad de la autodeclaración para las parejas homosexuales) para estimar qué porcentaje de ellas serían uniones estables. Sin embargo, teniendo en cuenta que el propósito principal de este trabajo pasa por la

3. Nota: de ahora en adelante, entenderemos por tipo de pareja el hecho de que ésta sea formada por dos cónyuges de distinto sexo (heterosexual) o del mismo sexo (homosexual).

4. Por autodeclaración, nos referimos a la posibilidad de identificar como cónyuge a una persona del mismo sexo, en la sección de relaciones de parentesco entre los miembros del hogar. 
descripción de la composición de las parejas homosexuales, más que por su cuantificación, hemos optado por trabajar exclusivamente con las uniones autodeclaradas en el censo de 2001.

El análisis de dichas parejas se beneficia de la riqueza de variables que el censo ofrece: la edad, el sexo, el estado civil, el nivel de instrucción, el lugar de residencia, la nacionalidad, etc. El interés reside en cruzar la información de ambos cónyuges para identificar las diferencias y las semejanzas entre ambos. El servidor de Internet del INE ofrece la posibilidad de cruzar esta información para la mayoría de variables, sin embargo, para las demás, es preciso recorrer a la muestra de microdatos del $5 \%$ de hogares (ver tabla 1 ). El servidor del INE informa, por defecto, de las características del «hombre generador del núcleo» y de las de la "mujer generadora del núcleo». En el caso de las parejas homosexuales, esta clasificación se mantiene intacta, de manera que uno de los cónyuges se convierte en mujer y el otro, en hombre necesariamente. Sin embargo, resulta interesante conocer el criterio utilizado por el INE para realizar tal asignación, puesto que no es arbitraria: es el cónyuge de mayor edad el que es considerado hombre y el de menor edad, mujer.

Podría pensarse que analizar la composición de las parejas a partir del censo o del registro de matrimonios influye sobre los resultados obtenidos, en la medida en que, en un caso, conocemos a las parejas en el momento del censo $y$, en el otro, en el de constituirse el matrimonio. La diferencia de observación entre la constitución de la unión y el momento censal puede, en efecto, introducir un sesgo significativo acorde con la antigüedad de la pareja. Sin embargo, en el caso de las parejas homosexuales en España, el mismo sesgo puede existir con los datos del MNP, puesto que los primeros matrimonios homosexuales que se celebran a partir de julio de 2005 corresponden a uniones de hecho que ya se habían constituido previamente y que se formalizan ahora con el cambio jurídico. Así, como no podemos asimilar el momento del matrimonio con el inicio de la unión, persiste el desfase temporal entre el momento de observación de la pareja y el momento de su constitución.

\section{Las uniones homosexuales en España}

En el censo de la población española de 1 de noviembre de 2001, 13.992 hombres declararon vivir en pareja estable con otro hombre, mientras 6.956 mujeres declararon tener a otra mujer como cónyuge. La tabla 2 nos muestra cual es la distribución territorial y por tipo de núcleo de dichas parejas, así como de las parejas heterosexuales. En primer lugar, las distribuciones muestran que el $72 \%$ de las parejas de mujeres y el $90 \%$ de las parejas de hombres no conviven con hijos, mientras que este porcentaje sólo representa el 32\% en el caso de las parejas heterosexuales (considerando a todas las edades en conjunto). Además de las diferencias en el tipo de núcleo por tipo de pareja, resulta interesante identificar las que se dan en las propias parejas homosexuales según el sexo de sus cónyuges. Las femeninas conviven con hijos en mayor medida que las masculinas. Esta diferencia en la composición del hogar y la posibilidad de 
Tabla 2. Distribución territorial y por tamaño del hogar según el tipo de pareja

\begin{tabular}{|c|c|c|c|c|}
\hline & $\begin{array}{l}\text { Parejas de } \\
\text { distinto sexo }\end{array}$ & $\begin{array}{c}\text { Parejas del } \\
\text { mismo sexo, } \\
\text { femenino }\end{array}$ & $\begin{array}{l}\text { Parejas del } \\
\text { mismo sexo, } \\
\text { masculino }\end{array}$ & $\begin{array}{l}\text { Ratio parejas } \\
\text { del mismo sexo/ } \\
\text { distinto sexo }\end{array}$ \\
\hline $\bar{N}$ & 9.500 .343 & 3.478 & 6.996 & \\
\hline \multicolumn{5}{|l|}{ Tipo de núcleo } \\
\hline Pareja sin hojos & $31,9 \%$ & $72,1 \%$ & $90,8 \%$ & \\
\hline Pareja con hijos & $68,1 \%$ & $27,9 \%$ & $9,2 \%$ & \\
\hline \multicolumn{5}{|l|}{ Ámbito } \\
\hline Zona urbana & $65,5 \%$ & $79,0 \%$ & $77,9 \%$ & \\
\hline Zona intermedia & $18,1 \%$ & $12,2 \%$ & $12,0 \%$ & \\
\hline \multirow[t]{2}{*}{ Zona rural } & $16,5 \%$ & $8,8 \%$ & $10,1 \%$ & \\
\hline & $100 \%$ & $100 \%$ & $100 \%$ & \\
\hline \multicolumn{5}{|l|}{ Comunidad Autónoma } \\
\hline Balears (Islas) & $2 \%$ & $4 \%$ & $5 \%$ & 2,2 \\
\hline Canarias & $4 \%$ & $7 \%$ & $7 \%$ & 2,0 \\
\hline Madrid (Comunidad de) & $13 \%$ & $24 \%$ & $24 \%$ & 2,0 \\
\hline Ceuta & $0 \%$ & $0 \%$ & $0 \%$ & 2,0 \\
\hline Melilla & $0 \%$ & $0 \%$ & $0 \%$ & 1,9 \\
\hline Cataluña & $16 \%$ & $24 \%$ & $23 \%$ & 1,6 \\
\hline Navarra (Comunidad Floral de) & $1 \%$ & $1 \%$ & $1 \%$ & 1,1 \\
\hline Comunidad Valenciana & $11 \%$ & $10 \%$ & $10 \%$ & 1,0 \\
\hline Murcia (Región de) & $3 \%$ & $2 \%$ & $3 \%$ & 0,9 \\
\hline País Vasco & $5 \%$ & $4 \%$ & $4 \%$ & 0,9 \\
\hline Andalucía & $18 \%$ & $11 \%$ & $12 \%$ & 0,7 \\
\hline Aragón & $3 \%$ & $1 \%$ & $2 \%$ & 0,7 \\
\hline Rioja (La) & $1 \%$ & $0 \%$ & $0 \%$ & 0,6 \\
\hline Cantabria & $1 \%$ & $1 \%$ & $1 \%$ & 0,5 \\
\hline Asturias (Principado de) & $3 \%$ & $1 \%$ & $1 \%$ & 0,5 \\
\hline Galicia & $7 \%$ & $3 \%$ & $3 \%$ & 0,5 \\
\hline Castilla-La Mancha & $4 \%$ & $2 \%$ & $2 \%$ & 0,4 \\
\hline Castilla y León & $6 \%$ & $2 \%$ & $2 \%$ & 0,4 \\
\hline \multirow[t]{2}{*}{ Extremadura } & $3 \%$ & $1 \%$ & $1 \%$ & 0,3 \\
\hline & $100 \%$ & $100 \%$ & $100 \%$ & \\
\hline
\end{tabular}

Fuente: censo de población 2001.

que haya descendencia estará vinculada también con las relaciones anteriores de los cónyuges, que se ven reflejadas parcialmente en su estado civil (en un momento, 2001, en el que las parejas homosexuales todavía no podían casarse). En la siguiente sección, veremos como la proporción de mujeres en unión homosexual alguna vez casadas (con un hombre, se entiende) es superior al de hombres alguna vez casados (tabla 3).

En segundo lugar, también la distribución por ámbito rural y urbano denota diferencias según el tipo de unión. Las parejas homosexuales aparecen más concentradas en las zonas urbanas (es decir, en municipios de más de 50.000 habitantes), con casi un $80 \%$, que las heterosexuales, con un $65 \%$. 
En tercer y último lugar, la tabla 2 nos informa de la distribución territorial de las parejas según el tipo. Si comparamos las distribuciones de cada uno, observamos como las parejas homosexuales están concentradas en Madrid y Cataluña, de acuerdo con la anteriormente observada concentración de estas parejas en zonas urbanas. En este sentido, Madrid y Cataluña acaparan relativamente más parejas homosexuales por el peso de las áreas metropolitanas de Madrid y Barcelona. Sin embargo, si leemos la ratio de parejas homosexuales sobre heterosexuales de cada comunidad, descubrimos que es en las Islas Baleares y en Canarias donde las parejas homosexuales tienen un peso relativo superior, alrededor de un $2 \%$. Precisamente, los cónyuges en unión homosexual que residen en estas dos comunidades autónomas insulares han nacido mayoritariamente fuera de ellas (el 79\% de los que residen en Baleares y el $66 \%$ de los que lo hacen en Canarias), cosa que no ocurre con los cónyuges en unión heterosexual. Estas dos zonas aparecen, así, como un destino residencial preferido de las parejas homosexuales. De todas formas, las diferencias entre comunidades son, a estos efectos, poco destacables y lo que se impone es el poco peso de dichas uniones, que no supera en ningún caso el 3\%o.

\section{Caracterización sociodemográfica de los cónyuges} (edad, sexo, nivel de instrucción, nacionalidad)

En la figura 1, se presenta, mediante dos pirámides de población superpuestas, la estructura por edad y sexo de la población de cónyuges residentes en España según el tipo de unión que forman, heterosexual o homosexual. En primer lugar, observamos claramente como los cónyuges que están unidos con un individuo del mismo sexo son más jóvenes que los que están unidos con uno del sexo opuesto. Esta diferencia en la estructura de edad debe ser leída en clave de generación, por lo que debe admitirse que los que están en unión homosexual son jóvenes porque las generaciones más antiguas entraron menos en uniones homosexuales, y no por efecto de edad, es decir, porque hayan salido de la unión a partir de cuando tenían determinados años.

En segundo lugar, observamos la clara diferencia de sexo en el número de cónyuges homosexuales. La constatación de que, a todas las edades, hay más hombres que mujeres coincide con los resultados empíricos obtenidos en otros países como Francia, Suecia o Noruega. La interpretación de dicha diferencia constituye un punto clave de la investigación del fenómeno de la homosexualidad, puesto que abre la puerta a la hipótesis de una incidencia diferencial por sexo de dicha orientación sexual. Sin embargo, algunos autores insisten en aclarar que no se trata aquí estrictamente del número de homosexuales, sino del número de uniones homosexuales, de tal modo que también deberían considerarse factores que pudieran motivar de modo diferencial a hombres y mujeres a entrar en unión homosexual, o bien a declarar que lo han hecho. Los autores también han señalado las diferencias en las motivaciones para formalizar las uniones (en aquellos casos posibles en que dicha formalización es legal). 


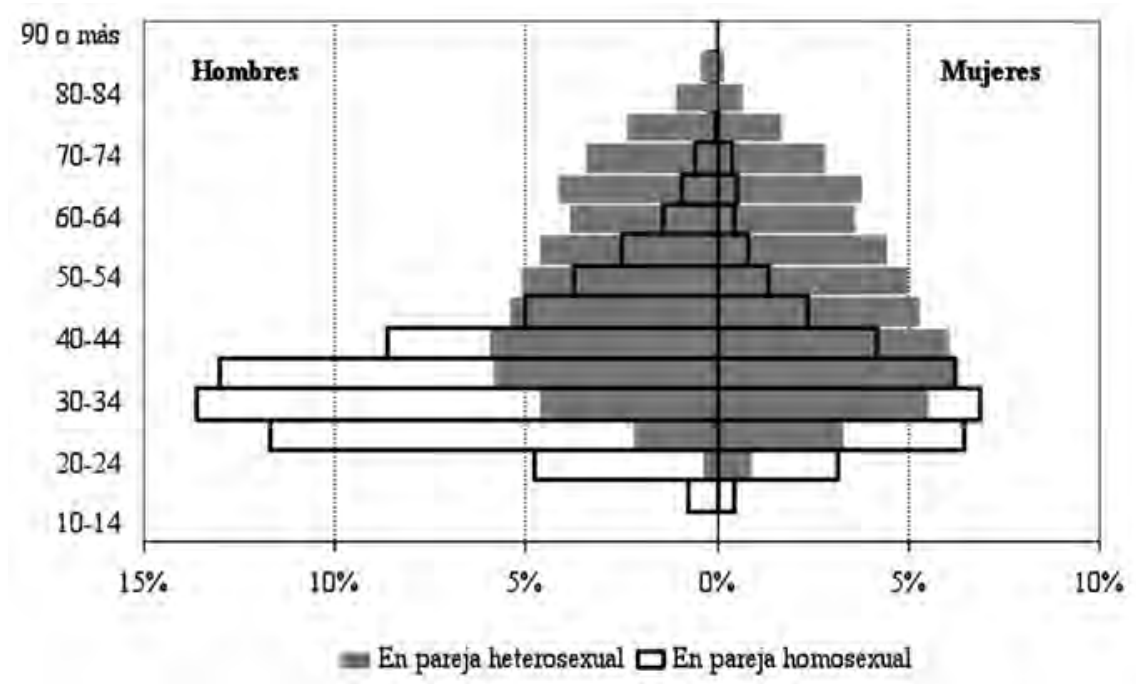

Figura 1. Pirámide de población de los cónyugues según el tipo de pareja. Fuente: censo de población 2001.

En este sentido, Andersson et al. (2006) destacan como factores que pueden llevar a los hombres a formalizar más sus uniones homosexuales que a las mujeres en Suecia y Noruega a la protección ante una mortalidad temprana provocada por el sida (de mayor incidencia masculina) o a la conveniencia de dar un estatus legal al cónyuge extranjero, en el caso de las uniones mixtas (más frecuentes entre hombres que entre mujeres).

Además de las diferencias por edad y sexo entre los cónyuges en unión homosexual y heterosexual, la tabla 3 nos presenta las diferencias en su composición por estado civil, nivel de instrucción y nacionalidad. En primer lugar, en relación con el estado civil, se aprecia el efecto de la no legalización de las uniones homosexuales en el hecho de que la mayoría de los cónyuges, a diferencia de los heterosexuales, son solteros ${ }^{5}$. Pero, dentro de esta tendencia, resulta interesante constatar las diferencias por sexo, puesto que las proporciones de solteros varían de un $83 \%$ para los hombres a un $72 \%$ para las mujeres. Esta diferencia de once puntos implica que las mujeres que están unidas con otra mujer han estado casadas anteriormente con un hombre, por lo menos en una ocasión, en mayor medida que los hombres que se encuentran en unión homosexual.

En segundo lugar, la estructura educativa de los cónyuges también presenta diferencias notables en función del tipo de unión. Los hombres y las mujeres en unión homosexual tienen mayor instrucción, de manera que se concen-

5. Tras la legalización del matrimonio homosexual en julio de 2005, será interesante estudiar el grado de institucionalización de las uniones. 
Tabla 3. Distribución por estado civil, nivel de instrucción y nacionalidad de los cónyuges según tipo de pareja

\begin{tabular}{|c|c|c|c|c|}
\hline & \multicolumn{2}{|c|}{$\begin{array}{l}\text { Cónyuges en pareja } \\
\text { de distintos sexo }\end{array}$} & \multicolumn{2}{|c|}{$\begin{array}{l}\text { Cónyuges en pareja } \\
\text { del mismo sexo }\end{array}$} \\
\hline & Hombres & Mujeres & Hombres & Mujeres \\
\hline $\bar{N}$ & 9.500 .343 & 9.500 .343 & 13.992 & 6.956 \\
\hline \multicolumn{5}{|l|}{ Estado civil } \\
\hline Soltero & 3,8 & 3,8 & 82,9 & 72,4 \\
\hline Casado & 94,8 & 94,6 & 9,5 & 13,2 \\
\hline Viudo & 0,2 & 0,4 & 0,8 & 3,2 \\
\hline Separado & 0,7 & 0,7 & 3,3 & 6,0 \\
\hline \multirow{2}{*}{ Divorciado } & 0,5 & 0,5 & 3,5 & 5,1 \\
\hline & 100 & 100 & 100 & 100 \\
\hline \multicolumn{5}{|l|}{ Nivel de distribución } \\
\hline Inferior a primaria & 16,1 & 17,4 & 6,0 & 6,4 \\
\hline Primaria & 26,3 & 27,3 & 14,2 & 12,9 \\
\hline Secundaria & 45,2 & 43,8 & 54,1 & 54,3 \\
\hline \multirow[t]{2}{*}{ Estudios superiores } & 12,5 & 11,5 & 25,7 & 26,4 \\
\hline & 100 & 100 & 100 & 100 \\
\hline \multicolumn{5}{|l|}{ Nacionalidad* } \\
\hline Extranjero/a & 2,8 & 3,0 & 26,6 & 24,6 \\
\hline \multirow[t]{2}{*}{ Español/a } & 97,2 & 97,2 & 73,4 & 75,4 \\
\hline & 100 & 100 & 100 & 100 \\
\hline
\end{tabular}

Fuente: censo de población 2001.

*La información sobre la nacionalidad ha sido obtenida a partir de la muestra de microdatos del $5 \%$ de los hogares, porque el INE no informa de esta variable en su servidor para el total de la población.

tra el $80 \%$ de sus efectivos en los niveles de educación secundaria y superior. No es éste el caso de los cónyuges con una pareja de distinto sexo, cuya estructura educativa es parecida a la del conjunto de la población española, con más de $25 \%$ de los individuos con estudios primarios y más de un $16 \%$ sin tan siquiera estudios primarios. Esta diferencia de instrucción ha sido sometida a una estandarización por edad, teniendo en cuenta que las diferencias en la estructura por edad observadas y la juventud de los cónyuges en unión homosexual podrían jugar a favor de su estructura educativa más favorable. Sin embargo, la estandarización no reduce apenas las diferencias educativas entre cónyuges de ambos tipos de unión, lo que significa que, efectivamente, los individuos que forman pareja con una persona de su mismo sexo poseen un nivel más elevado de instrucción, sin que ello tenga que ver con su mayor juventud.

Finalmente, la caracterización de los cónyuges se completa por su nacionalidad. Mientras que apenas el 3\% de los cónyuges con pareja de distinto sexo es extranjero, esta proporción alcanza el $25 \%$ de los que tienen pareja del mismo sexo. Es decir que el peso de los extranjeros, tanto hombres como mujeres, en las uniones homosexuales es mayor que en el del conjunto de las pare- 
Tabla 4. Características individuales asociadas al hecho de ser miembro de una pareja homosexual (parámetros estimados de un modelo de regresión logística)

\begin{tabular}{|c|c|c|c|}
\hline & $\mathrm{N}$ & B & $\operatorname{Exp}(B)$ \\
\hline \multicolumn{4}{|l|}{ Sexo } \\
\hline Hombre (ref.) & 475.689 & & \\
\hline Mujer & 475.356 & 0,61 & $0,54^{* *}$ \\
\hline \multicolumn{4}{|l|}{ Edad } \\
\hline $15-29$ (ref.) & 66.073 & & ** \\
\hline $30-44$ & 328.278 & 0,55 & $1,73^{* *}$ \\
\hline$>=45$ & 556.694 & 0,36 & $1,43^{* *}$ \\
\hline \multicolumn{4}{|l|}{ Estado civil } \\
\hline Soltero (ref.) & 37.172 & & ** \\
\hline Casado & 899.613 & $-4,46$ & $0,01^{* *}$ \\
\hline Viudo & 2.865 & $-1,37$ & $0,25^{* *}$ \\
\hline Separado/Divordiado & 11.395 & $-0,72$ & $0,49^{* *}$ \\
\hline \multicolumn{4}{|l|}{ Nivel de instrucción } \\
\hline Inferior a obligatorio (ref.) & 413.437 & & ** \\
\hline Obligatorio & 260.473 & 0,00 & $1,00-$ \\
\hline Secundaria & 162.594 & 0,11 & $1,11-$ \\
\hline Estudios superiores & 114.541 & 0,30 & $1,35^{* *}$ \\
\hline \multicolumn{4}{|l|}{ Nacionalidad } \\
\hline Extranjero/a & 2,8 & 3,0 & 26,6 \\
\hline \multicolumn{4}{|l|}{ Nacionalidad* } \\
\hline Extranjero (ref.) & 28.083 & 3,0 & 26,6 \\
\hline Español/a & 922.962 & $-1,12$ & $0,33^{* *}$ \\
\hline \multicolumn{4}{|l|}{ Tipo de núcleo } \\
\hline Pareja sin hijos (ref.) & 284.539 & & \\
\hline Pareja con hijos & 666.506 & $-2,11$ & $0,12^{* *}$ \\
\hline Constante & & $-2,68$ & $0,07^{* *}$ \\
\hline
\end{tabular}

**Significativo con un intervalo de confianza de un $95 \%$.

jas. En el siguiente apartado, veremos como se traduce esta mayor presencia en la composición de las uniones en términos de endogamia.

La estandarización por edad nos ha permitido confirmar la particular estructura educativa de los cónyuges homosexuales. Sin embargo, este ejercicio de estandarización resulta parcial y por ello aplicamos una regresión multivariada, en concreto, un modelo de regresión logística. Con este modelo, podemos estimar en forma de parámetros $(\exp ())$ el efecto neto de cada una de las variables explicativas incluidas en el modelo sobre la variable independiente, en nuestro caso, estar en unión homosexual en comparación con estar en unión heterosexual. Cuando el valor del parámetro $\exp ()$ es igual a 1, la probabilidad de estar en unión homosexual es igual a la de estar en unión heterosexual, de manera que la característica tiene un efecto neutro. Cuando el parámetro toma valores superiores a 1 , la probabilidad se incrementa, y cuando toma valores fraccionados, entre 0 y 1 , la probabilidad se reduce. La tabla 4 presenta los 
resultados obtenidos que vienen a confirmar la caracterización sociodemográfica de los cónyuges obtenida hasta hora. En primer lugar, en el 2001, la probabilidad de las mujeres a estar en unión homosexual es prácticamente la mitad que la de los hombres. En segundo lugar, esta probabilidad es muy elevada en las edades adultas y es mucho más baja para cualquier estado civil que para los solteros. En lo que se refiere a la educación, controlando por el resto de variables, podemos afirmar que la probabilidad de estar en unión es un 35\% más elevada para los que tienen estudios superiores que para los que no han terminado la enseñanza obligatoria. Finalmente, se confirma que los españoles tienen menor probabilidad de estar unidos con alguien del mismo sexo que los extranjeros (a sexo, edad, estado civil y nivel de instrucción iguales) y que esta probabilidad disminuye en los núcleos con hijos.

\section{Composición de las uniones (edad, nivel de instrucción, nacionalidad)}

La diferencia media de edad entre los cónyuges que contraen matrimonio ha disminuido en el último cuarto de siglo, puesto que ha pasado de 2,8 a 2,3 años entre la edad de los maridos y las esposas. Si bien el censo de 2001 no permite captar dicha evolución, sí muestra como la mayoría de las parejas heterosexuales (incluyendo a todas las generaciones de matrimonios y también a las parejas de hecho) tienen una diferencia de edad de entre 1 y 4 años, mientras que las uniones con una diferencia extrema de 10 años o más son claramente minoritarias (ver tabla 5). La comparación con la diferencia de edad entre cónyuges de uniones homosexuales desvela que, en su caso, las parejas con una diferencia igual o superior a los 10 años supone casi un tercio de las parejas masculinas y casi un cuarto de las femeninas. La diferencia entre hombres y mujeres es consistente con la registrada en otros países como Suecia y Noruega, donde las uniones homosexuales masculinas presentan mayores disparidades de edad que las femeninas y éstas que las heterosexuales (Andersson et al., 2006). Las uniones homosexuales donde ambos tienen la misma edad, por el contrario, son claramente minoritarias. La tabla 4 nos muestra también la proporción de uniones endógamas (aquéllas donde ambos cónyuges tienen la misma nacionalidad) según el tipo de unión. El importante peso de los extranjeros entre los cónyuges homosexuales se traduce en una proporción de exogamia más elevada en las uniones homosexuales que en las heterosexuales. Es decir, estos numerosos extranjeros no forman pareja entre sí, sino que tienden más bien a unirse con españoles, especialmente en el caso de los hombres ${ }^{6}$.

Finalmente, la tabla 6 presenta las proporciones de parejas para cada combinación de nivel de instrucción. En el caso de las parejas de distinto sexo, se

6. Los niveles de exogamia de los matrimonios contraídos entre 2005 y 2008 son también mucho más elevados en los matrimonios del mismo sexo que de distinto: en concreto un 37\% de los de hombres y un $20 \%$ de los de mujeres son entre español/a y extranjero/a. La homogamia por edad de estos matrimonios también es inferior a la de los de distinto sexo: la diferencia media de edad entre cónyuges es de 8 años para los hombres y de 5 para las mujeres. 
Tabla 5. Composición por edad y nacionalidad de las uniones según el tipo de que se trate

\begin{tabular}{lccc}
\hline & Parejas de & \multicolumn{2}{c}{$\begin{array}{c}\text { Parejas del } \\
\text { mismo sexo }\end{array}$} \\
\cline { 2 - 4 } & distinto sexo & Masculinas & Femeninas \\
\hline Diferencia de edad (en años) & & $6,5 \%$ & $8,3 \%$ \\
0 & $10,4 \%$ & $37,3 \%$ & $42,1 \%$ \\
$1-4$ & $61,5 \%$ & $26,3 \%$ & $26,5 \%$ \\
$5-9$ & $23,1 \%$ & $30,0 \%$ & $23,0 \%$ \\
$10+$ & $5 \%$ & $100 \%$ & $100 \%$ \\
\hline Nacionalidad* & $100 \%$ & & \\
Exogamia & & $17 \%$ & $10 \%$ \\
Endogamia & $2 \%$ & $83 \%$ & $90 \%$ \\
& $98 \%$ & $100 \%$ & $100 \%$ \\
\hline
\end{tabular}

Fuente: censo de población 2001.

* Nota: las proporciones de endogamia y exogamia han sido obtenidas a partir de la muestra de microdatos del 5\% de hogares, porque el INE no informa en su servidor de la nacionalidad de los cónyuges para el total de la población.

combina el nivel del hombre con el de la mujer, de tal manera que es posible medir la homogamia adicionando las cuatro proporciones que se encuentran en la diagonal de la tabla, como también la heterogamia, comparando las proporciones de la parte superior (en la que los hombres tienen menos estudios que las mujeres) con la parte inferior (en la que son ellas las menos instruidas). En el caso de las parejas del mismo sexo, la medición de la homogamia sigue teniendo su interés, pero el análisis de las distintas pautas de heterogamia por sexo deja de tener sentido. Por exigencia de la base de datos del INE, en las parejas homosexuales comparamos el nivel de instrucción del cónyuge de más años con el de menos años, de modo que, si no se busca una relación entre la edad y la selección de la pareja, la pertinencia del análisis de simetría desaparece. Centrándonos, pues, en los niveles de homogamia, constatamos como éstos son más elevados en las parejas de distinto sexo, donde las parejas del mismo nivel de instrucción suponen un $70 \%$ del total que en las del mismo sexo, donde representan un 58\% y un 57\% para hombres y mujeres, respectivamente (tabla 6$)^{7}$. Esta diferencia, que está en la misma línea que las disparidades observadas en términos de edad y de nacionalidad, puede relacionarse con la estructura educativa de los cónyuges. Si la estructura educativa de los cónyuges en unión homosexual fuera más homogénea que la de los heterosexuales, este efecto de estructura podría explicar el menor nivel de homogamia. Con el fin de descartar tal efecto, utilizamos un indicador de homogamia ${ }^{8}$ que

7. Estos porcentajes de homogamia total se obtienen por adición de las celas de la diagonal de la tabla 6.

8. Dicho indicador está desarrollado y aplicado al estudio de la homogamia educativa en Noruega por Birkelund y Heldal (2003). 
Tabla 6. Composición por nivel de instrucción de las uniones según el tipo de que se trate

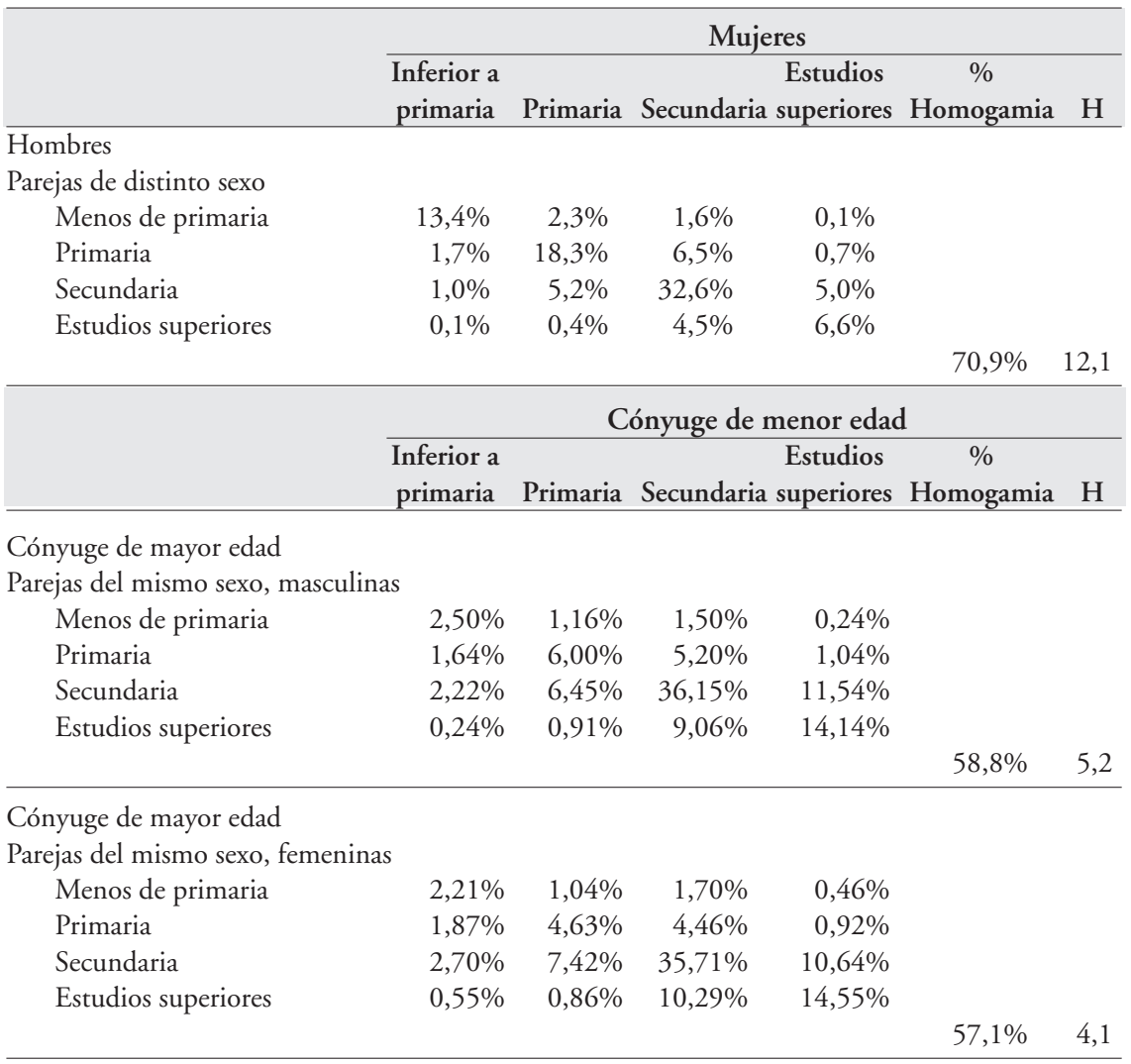

Fuente: censo de población 2001.

Nota: la distribución de las uniones en cada matriz está calculada respecto al total de uniones de la matriz.

controla específicamente, como si estandarizara, las diferencias en la estructura educativa. Cuando el indicador $\mathrm{H}$ es igual a 1 , expresa que no hay relación alguna entre el nivel educativo de los cónyuges, mientras que los valores positivos indican una interacción matrimonial en las combinaciones homógamas más intensa de la esperada de acuerdo con la distribución educativa de los cónyuges, es decir, una preferencia neta por los cónyuges de igual nivel educativo. La tabla 6 muestra que $\mathrm{H}$ adquiere valores claramente superiores tanto para las parejas heterosexuales como para las homosexuales y confirma que, a igual estructura educativa, las parejas homosexuales masculinas son dos veces menos homógamas que las heterosexuales, y las femeninas, tres veces menos.

Hemos descartado el efecto de la estructura educativa de los cónyuges sobre el nivel de homogamia educativa de las parejas. Sin embargo, también es inte- 
Tabla 7. Características de la pareja asociadas al tipo de unión (homosexual/ heterosexual) (parámetros estimados de un modelo de regresión logística)

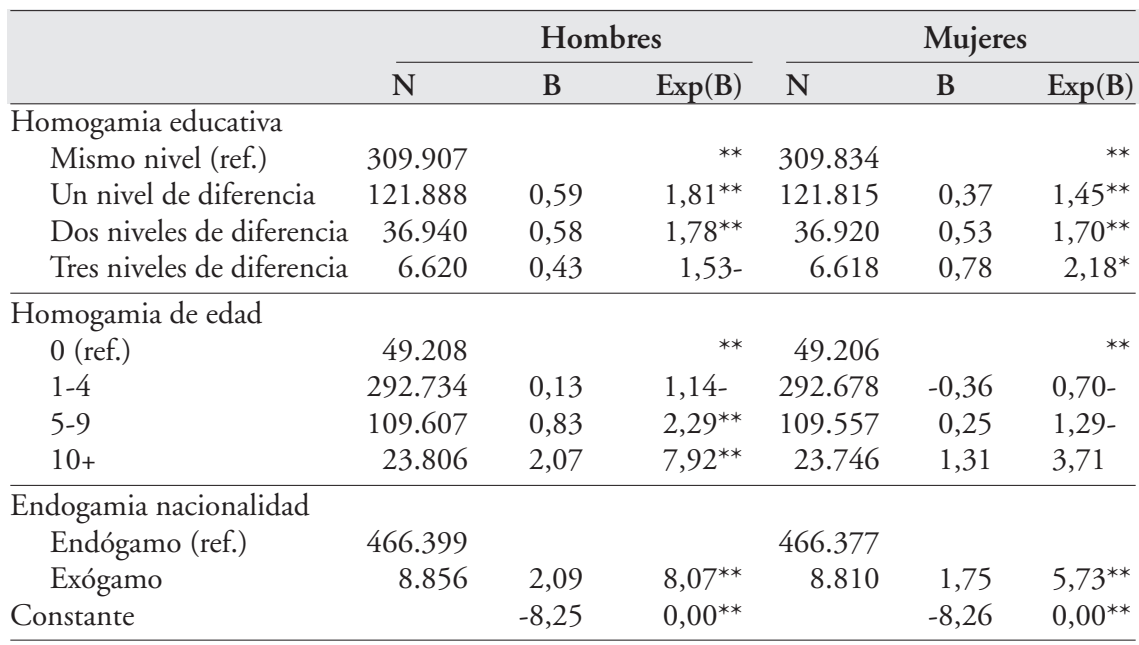

**Significativo con un intervalo de confianza de un $95 \%$, *intervalo de confianza de un $90 \%$.

resante descartar el efecto que la composición en relación con otras variables puede tener sobre la composición por nivel de instrucción. Es decir, si sabemos que la disparidad de edades y la endogamia son mayores en las parejas homosexuales que en las heterosexuales, ¿en qué medida pueden estas diferencias aumentar la probabilidad de que haya disparidades educativas entre los cónyuges? ¿Y viceversa? Los resultados de la regresión multivariable presentados en la tabla 7 muestran como se asocian las tres dimensiones de la heterogamia de las parejas (educativa, de edad y por nacionalidad), para hombres y mujeres, a la probabilidad de estar en unión homosexual, según el censo de 2001. En relación con las uniones de composición homógama (que figuran como categoría de referencia), las uniones con un cónyuge más instruido que el otro, mayor que el otro y de una nacionalidad distinta presentan valores superiores a 1, lo que significa que la heterogamia está asociada positivamente con el tipo de unión homosexual. Por ejemplo, los hombres que presentan una diferencia de diez años o más con su pareja tienen casi ocho veces más posibilidades de estar en unión homosexual que heterosexual. Lo mismo ocurre para los hombres que están unidos con un cónyuge de distinta nacionalidad a la suya. En el caso de las mujeres, la pauta se mantiene, si bien los parámetros no alcanzan valores tan elevados. Podemos concluir que, aunque puedan reforzarse entre sí, cada una de las dimensiones de la composición de las parejas aquí analizadas está directa y positivamente relacionada con el tipo de unión homosexual, es decir que, en el año 2001, las uniones homosexuales eran más heterógamas en lo que a edad, nivel de instrucción y nacionalidad se refiere. 


\section{Conclusiones}

Ante la reciente legalización del matrimonio homosexual en España, y en un contexto europeo de expansión de los derechos de las parejas del mismo sexo, hemos constatado como este proceso abre también la vía para el estudio de estas parejas a partir de nuevas fuentes estadísticas: los registros de uniones y de matrimonios, el MNP en el caso español. Disponiendo todavía sólo de datos de matrimonios de 3 años completos, hemos caracterizado a los homosexuales que viven en pareja y sus uniones a partir de los datos del censo de 2001. Asumiendo que, a pesar de estar subregistradas, las parejas homosexuales autodeclaradas en el censo son representativas del conjunto, realizamos una exploración de sus características que resulta novedosa en un contexto de muy escasa investigación científica sobre la materia en España y que permite establecer la comparación con exploraciones análogas en países europeos.

Las uniones formadas por dos personas del mismo sexo en el año 2001 están formadas por cónyuges que se caracterizan por ser jóvenes, por poseer un elevado nivel de instrucción y por residir mayoritariamente en áreas urbanas. Estos tres elementos (generación, nivel socioeconómico y territorio) apuntan a la existencia en España, todavía, de ámbitos más o menos favorables donde llevar a cabo un proyecto de pareja homosexual. El número de uniones de hombres es dos veces mayor que el de mujeres, elemento que refuerza las tesis que apuntan a una mayor incidencia de la homogamia masculina que femenina, pero que no resulta determinante, puesto que puede existir un diferencial en la intensidad de entrada en unión corresidente.

En lo que se refiere a la composición de dichas uniones, es decir, al análisis comparado de las características de los cónyuges, los resultados apuntan a una mayor heterogamia que en las parejas heterosexuales en variables tan fundamentales como la edad, la nacionalidad o el nivel de instrucción. Esta heterogamia de las uniones homosexuales ha sido puesta de manifiesto en investigaciones empíricas en países como Francia, Suecia o Noruega y requiere de más investigación para poder encontrar elementos de interpretación que vinculen la polaridad en las características de los cónyuges que tienen el mismo sexo. Entre ellos, será preciso considerar si existen un funcionamiento y unas condiciones estructurales específicas del mercado matrimonial para las parejas homosexuales. En efecto, para parejas heterosexuales, las investigaciones han puesto de manifiesto que la oferta absoluta de candidatos, o bien relativa de candidatos de determinadas características, condicionan en buena medida la composición de las uniones resultantes. Más adelante, puede resultar interesante plantear las semejanzas y las diferencias de la estructura y la dinámica del mercado en el proceso de formación de cada tipo de pareja. 


\section{Referencias bibliográficas}

Andersson, G. et al. (2006). «The demographics of same-sex marriages in Norway and Sweden». Demography, 43 (1), 79-98.

Birkelund, G.E. y Heldal, J. (2003). «Who marries whom? Educational Homogamy in Norway». Demographic Research, 8 (1), 1-30.

BLACK, D. et al. (2000). "Demographics of the gay and lesbian population in the United States: Evidence from available systematic data sources». Demography, 37 (2), 139-154.

Brown, M. y KnOpp, L. (2006). «Places or polygons? Governmentality, scale, and the census in The Gay and Lesbian Atlas». Population, Space and Place, 12, 223242.

DomingO, A. (1997). La formación de la pareja en tiempos de crisis: Madrid y Barcelona, 1975-1995. Universidad Nacional a Distancia, Departamento de Sociologia II. Tesis doctoral.

Esteve, A.; Cortina, C. y Cabré, A. (2009). «Long Term Trends in Age-Assortative Mating: Spain, 1922-2006». Population, 64(1): 173-202.

Esteve, A. y Cortina, C. (2007). «Con quién se emparejan los españoles? Homogamia conyugal por edad, nivel de instrucción y origen geográfico». En: CABRÉ et al. Constitución familiar en España. Bilbao: Fundación Banco Bilbao Vizcaya, 93-138.

Esteve, A. y CorTinA, C. (2006). "Changes in educational assortative mating in contemporary Spain». Demographic Research, 14 (18), 429-452.

Festy, P. (2006). "La legalisation des couples homosexuels en Europe». Population, 61(4) : 493-531.

Festy, P. y Digoix, M. (eds.) (2004). «Same-sex couples, same-sex partnerships \& homosexual marriages: A focus on cross-national differentials». Documents de Travail de l'INED, 124.

Festy, P.; Digoix, M. y GARnier, B. (2004). «What if same-sex couples exist in France after all?». En: FESTY, P. y DigOIX, M. (eds.). Same-sex couples, same-sex partnerships \& homosexual marriages: A focus on cross-national differentials. París: INED, 193209. Documents de Travail; 124.

JEPSEN, L. y JEPSEN, C. (2002). "An empirical analysis of the matching patterns of same-sex and opposite-sex couples». Demography, 39, 435-453.

LEY 13/2005, de 1 de julio, por la que se modifica el Código Civil en materia de derecho a contraer matrimonio.

Pichardo Galán, J.I. (2004). «Same-sex couples in Spain. Historical, contextual and symbolic factors». En: FESTY, P. y DigOIX, M. (eds.). Same-sex couples, same-sex partnerships \& homosexual marriages: A focus on cross-national differentials. París: INED, 159-174. Documents de Travail; 124.

WAALDIJK, W. (2005). «More or less together: Levels of legal consequences of marriage, cohabitation and registered partnership for different-sex and same-sex partners». Documents de Travail de l'INED, 125, 203. 